\title{
Erratum to: The two-loop energy-momentum tensor within the gradient-flow formalism
}

\author{
Robert V. Harlander ${ }^{\mathrm{a}}$, Yannick Kluth ${ }^{\mathrm{b}}$, Fabian Lange ${ }^{\mathrm{c}} \mathbb{C}$ \\ Institute for Theoretical Particle Physics and Cosmology, RWTH Aachen University, 52056 Aachen, Germany
}

Received: 13 September 2019 / Accepted: 19 September 2019 / Published online: 18 October 2019

(c) The Author(s) 2019

Erratum to: Eur. Phys. J. C (2018) 78:944

https://doi.org/10.1140/epjc/s10052-018-6415-7

Two typos in [1] are corrected.

- The left-hand side of Eq. (43) should read $Z_{\chi}^{-1}$ instead of $Z_{\chi}$.

- The sign of the first term of the coefficient $\gamma_{1,32}$ in Eq. (63) should be reversed. The correct coefficient reads

$$
\gamma_{1,32}=-\frac{272}{27} C_{A} T_{F}-\frac{392}{27} C_{F} T_{F} .
$$

Ancillary files for the paper can be found at arXiv:1808.09837.
Acknowledgements We are indebted to Mauro Papinutto for pointing out the typo in Eq. (43).

Open Access This article is distributed under the terms of the Creative Commons Attribution 4.0 International License (http://creativecomm ons.org/licenses/by/4.0/), which permits unrestricted use, distribution, and reproduction in any medium, provided you give appropriate credit to the original author(s) and the source, provide a link to the Creative Commons license, and indicate if changes were made. Funded by SCOAP S $^{3}$

\section{Reference}

1. R.V. Harlander, Y. Kluth, F. Lange, The two-loop energy-momentum tensor within the gradient-flow formalism. Eur. Phys. J. C 78, 944 (2018). arXiv:1808.09837 [hep-lat]

The original article can be found online at https://doi.org/10.1140/ epjc/s10052-018-6415-7.

\footnotetext{
a e-mail: harlander@physik.rwth-aachen.de

b e-mail: kluth@physik.rwth-aachen.de

c e-mail: flange@physik.rwth-aachen.de
} 